\title{
BIO-INSPIRED ADHESION DEVICES
}

\author{
E. Arzt and A. del Campo
}

\author{
${ }^{1}$ INM - Leibniz Institute for New Materials, Saarbrücken, Germany
}

\begin{abstract}
Adhesive joining with molecular (van der Waals) interactions without chemical glue is presently receiving much attention because of many potential applications. Research on how insects, spiders and geckos stick to surfaces has inspired a new paradigm: fibrillar surfaces with appropriate design can show much higher adhesion performance than flat surfaces. The insight gained in studying biological systems can be transferred to the development of optimized artificial attachment devices. By systematic variations of fiber diameter, aspect ratio and contact shape, we have produced, on a laboratory scale, artificial structures with adhesion strengths similar to the gecko. Further advances with switchable adhesion ("smart adhesives") have been demonstrated and may lead to interesting applications in medical products, sports good, construction materials and microfabrication.
\end{abstract}

\section{INTRODUCTION}

A new paradigm has emerged over the last years in adhesion: enhanced sticking of patterned versus planar surfaces. This concept of "contact splitting" [1] arises from the observation of flies, spiders and geckos, which exhibit attachment organs with long micro to nano-sized hairs, e.g. [2, 3]. The complex design of these hairs seems to be the key to their smart adhesion behavior, which enables repeated attachment and easy release from almost any kind of surface.
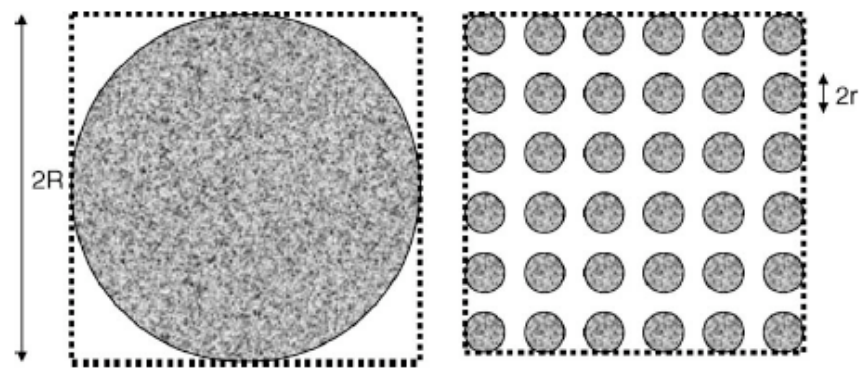

Figure 1: The principle of contact splitting: splitting of a reversible van der Waals contact (initially of radius $R$ ) into many fine contacts (new radii $r$ ) increases the adhesion force. This principle suggests the application of miniaturization technologies to achieve artificial adhesives.

Inspired by this principle, theory and experiment of reversible contact mechanics in small structures have made much progress in recent years, e.g. [4-16]. Yet attempts to produce such surfaces with significantly enhanced adhesion have so far met with mixed success. While individual fibers exhibited the expected attachment, larger surfaces often did not demonstrate superior adhesion performance. The reason for this failure very likely lies in the complexity of the problem: the design parameters and underlying adhesion mechanisms need to be understood and optimized on the nano, micro and macro scale. For this purpose, singular experiments, as are frequently published in the literature, fail to advance the subject; rather, systematic model studies backed by suitable modelling efforts are required. The present contribution describes some of our recent progress in this area.

\section{MICROPATTERNED MODEL SURFACES: EFFECTS OF FIBER SIZE AND SHAPE}

We have fabricated model fibrillar structures by soft-molding elastomeric precursors on photolithographic molds [17]. Typical structures, with $20 \mu \mathrm{m}$ diameter, are shown in Figure 2. Adhesion studies on regular arrays of Poly(dimethylsiloxane) (PDMS) micropillars (pillar radii were varied between 2.5 and $25 \mu \mathrm{m}$ and aspect ratio between 1 and 4) have proved the enhanced adhesion of structured surfaces against flat controls. Systematic studies of the influence of the contact radius and aspect ratio of the pillars on the final adhesion performance have demonstrated that decreasing the contact radius and increasing the aspect ratio of the pillars enhances adhesion [18].
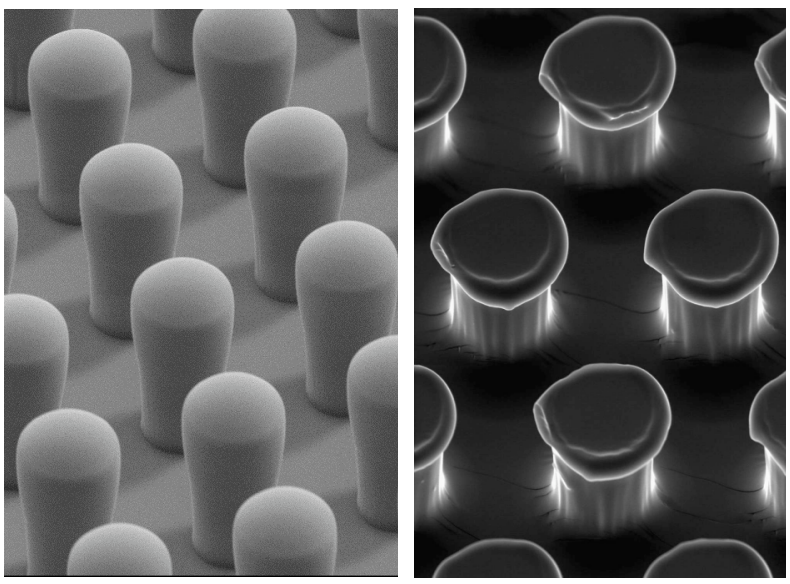

Figure 2: Micropatterned fibrillar PDMS surfaces: spherical tips (left) vs. mushroom-shaped tips (right). The diameter of both types of fibers is $20 \mu \mathrm{m}$. Adhesion is strongly enhanced by the presence of mushroom tips, which reach the adhesion strength of real gecko toes. (after del Campo et al. [21])

New methods for the fabrication of structured polymer surfaces possessing pillars with controlled 3D tip geometries were developed: spherical, spatula-like and suction cups [19, 20]. The fabrication strategies exploit the filling mechanism of lithographic templates by viscous polymers, combined with inking and printing steps using elastomeric precursors with various viscosities and crosslinking kinetics. Homogeneously structured areas were obtained which allow reproducible and reliable testing of adhesion behaviour and can be up scaled to prototypes. These fabrication routes do not require sophisticated 3D structuring equipment and can be extended to other materials, dimensions and geometries.

Our adhesion experiments show significant variation of the adhesion performance of the surfaces depending on the tip geometry and testing conditions [21]. The highest pull-off forces were found for mushroom-like pillars, which showed an increase of up to 30 times over the flat controls (see Fig. 2b and Fig. 3). This corresponds roughly to the adhesion strength of a gecko toe. Flat and spherical shapes showed lower adhesion, while concave shapes resulted in the poorest performance, coupled with an unusual preload dependence.

The fiber radius effect studied previously augments the shape effect, creating vastly different "splitting efficiencies" [5] for the 
different shapes. Within the size range tested, mushroom and spatular shapes hold the greatest promise for further adhesion improvement through size reduction.

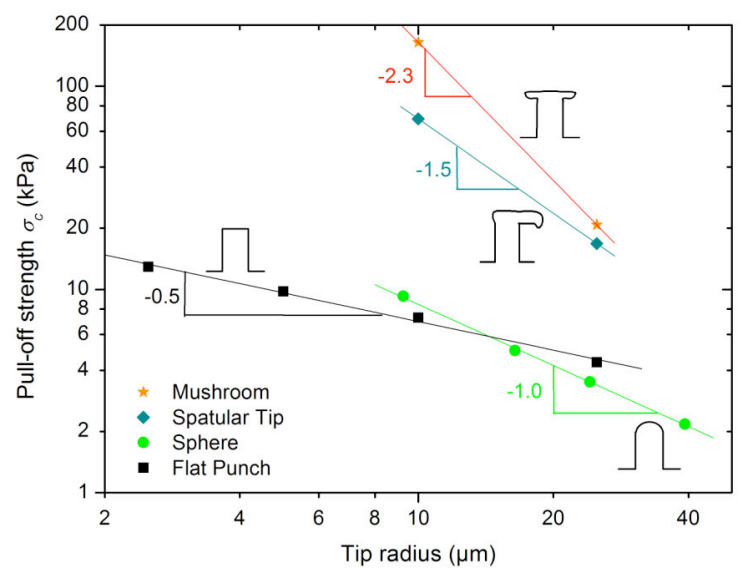

Figure 3: Adhesion strength (pull-off force divided by apparent contact area) of different contact shapes as a function of fiber radius. Mushroom and spatular tips scale most favorably to smaller dimensions. (after [21])

\section{TOWARDS SWITCHABLE ADHESION: "SMART" SURFACES}

We have recently demonstrated that patterning technologies can be combined with responsive polymer materials to create microstructured surfaces with switchable adherence [22]. Application of an external field (e.g. temperature) causes changes in the topographical design and this influences the final adhesion performance. A shape memory polymer was selected for this purpose. Arrays of microfibers, with diameters between 0.5 and 50 $\mu \mathrm{m}$ and lengths between 10 and $100 \mu \mathrm{m}$, were patterned by soft molding. Mechanical deformation at the shape-memory transition temperature, followed by cooling to room temperature in the deformed position yielded a temporary non-adhesive surface consisting of pillars in a tilted position. By reheating above the transition temperature, the patterned surface switches from the temporary non-adhesive state to a permanent adhesive surface with at least a 200 -fold increase in adherence. Such active structures may have interesting applications in responsive systems where adhesion or friction management is required.

\section{OUTLOOK}

While some of the ingredients of biological attachment systems have been successfully emulated in artificial systems, several challenges still lie ahead. Obviously, biological devices are much more complex from a geometrical and materials point of view. These peculiarities still need to be analyzed theoretically; for example, the superiority of mushroom-type fibers has recently been explained by an improvement of the near-perimeter stress distribution by numerical methods [23]. The next challenge is to imitate the hierarchical structure of biological systems in order to achieve good adhesion to rough surfaces; a first attempt has been made [14], but the resulting adhesion strengths need to be improved to be of practical use. Biological fibers are most likely composed of gradient materials, e.g. to allow small fiber radii without the propensity to clumping [9]. Also asymmetric and anisotropic contact shapes, as they are present in flies and geckos, will need to be reproduced to combine reliable adhesion with easy detachment. Because of the large parameter space, experimental works needs to be accompanied by suitable modelling activities.
For example, the concept of "adhesion design maps" [9], which are useful graphical displays of the trade-offs in producing efficient attachment structures, need to be extended from simple spherical to more complex shapes [24]. Finally, of course, practical applicability will depend on cost-effective fabrication routes for large areas; such developments are currently underway [25].

\section{REFERENCES}

[1] E. Arzt, S. Gorb and R. Spolenak, "From micro to nano contacts in biological attachment devices", PNAS 100 (19), 10603 10606 (2003)

[2] S. Gorb, "Attachment Devices of Insect Cuticle", Springer Netherland, 2001

[3] K. Autumn et al., "Adhesive force of a single gecko foot-hair", Nature 405, 681-685 (2000)

[4] E. Arzt, "Biological and artificial attachment devices: lessons for materials scientists from flies and geckos", Mater. Sci. Eng. C 26, 1245-1250 (2006)

[5] R. Spolenak, S. Gorb, H. Gao and E. Arzt, "Effects of contact shape on the scaling of biological attachments", PRSL A 461 (2054), 305-319 (2005)

[6] B.N.J. Persson, "On the mechanism of adhesion in biological systems", J. Chem. Phys. 118, 7614-7621 (2003)

[7] C.Y. Hui et al., J. Adhesion 81 (7-8), 699-721 (2005)

[8] L. Ma, R. McMeeking and E. Arzt, Int. J. Mats. Res. 98, 11561162 (2007)

[9] R. Spolenak, S. Gorb and E. Arzt, "Adhesion design maps for bio-inspired attachment systems", Acta Biomater. 1, 5-13 (2005)

[10] H. Yao and H. Gao, J. Mech. Phys. Solids 54 (6), 1120-1146 (2006)

[11] A.K. Geim et al., Nature Mat. 2 (7), 461-463 (2003)

[12] A. Ghatak et al., PRSL A 460, 2725-2735 (2004)

[13] J.Y. Chung and M.K. Chaudhury, JRS Interface 2 (2), 55-61 (2005)

[14] M.T. Northen and K.L. Turner, Nanotechnology 16, 11591166 (2005)

[15] M.Sitti and R.S. Fearing, J. Adh. Sci. Tech. 17 (8), 1055-1073 (2003)

[16] A.J. Crosby et al., Langmuir 21 (25), 11738-11743 (2005)

[17] A. del Campo and E. Arzt, „Design parameters and current fabrication approaches for developing bioinspired dry adhesives", Macromolecular Bioscience 7, 118-127 (2007)

[18] C. Greiner, A. del Campo and E. Arzt, "Adhesion of bioinspired micro-patterned surfaces: effects of pillar radius, aspect ratio and preload", Langmuir, 23, 3495-3502 (2007)

[19] A. del Campo, I. Alvarez, S. Filipe and M. Wilhelm, Advanced Functional Materials 17, 3590 (2007)

[20] A. del Campo, C. Greiner, I. Alvarez and E. Arzt, „Patterend surfaces with pillars with controlled and 3D tip geometry mimicking bioattachment devices“, Advanced Materials 19, 1973 (2007)

[21] A. del Campo, C. Greiner and E. Arzt, "Contact shape controls adhesion of bioinspired fibrillar surfaces", Langmuir 23, 10235-10243 (2007)

[22] S. Reddy, E. Arzt and A. del Campo, "Bioinspired surfaces with switchable adhesion made of shape memory polymers", $A d v$. Mats. 19, 3833-3837 (2007)

[23] A.V. Spuskanyuk, R.M. McMeeking, V.S. Deshpande and E. Arzt, "The effect of shape on the adhesion of fibrillar surfaces", Acta Biomaterialia, in press

[24] C. Greiner, R. Spolenak and E. Arzt, "Design Maps for NonSpherical Contact Shapes", in preparation

[25] A. Altherr, P. Oliveira and E. Arzt, unpublished. 